\title{
Hadronic matrix elements for exclusive rare $B$-meson decays
}

\author{
A. Khodjamirian* and Y.-M. Wang \\ Theoretische Physik 1, Naturwissenschaftlich-Technische Fakultät, \\ Universität Siegen, D-57068 Siegen, Germany \\ E-mail: khodjamehep.physik.uni-siegen.de, \\ wang@hep.physik.uni-siegen.de
}

\begin{abstract}
We report on the QCD calculation of the hadronic matrix elements relevant for the exclusive rare $B$ decays, such as $B \rightarrow K^{(*)} \ell^{+} \ell^{-}$and $B \rightarrow K^{*} \gamma$. The hadronic input for the decay observables, in addition to the heavy-light form factors, contains specific contributions, generated by the fourquark and penguin operators, such as the charm-loop effects. The corresponding hadronic matrix elements are calculated by the same method as the form factors, applying OPE and light-cone sum rules in QCD. This technique allows one to take into account the nonfactorizable soft-gluon contributions. The results are expressed in terms of (process-dependent) corrections to the shortdistance coefficients of the effective Hamiltonian. The impact of these corrections on the most important observables, e.g., on the forward-backward asymmetry in $B \rightarrow K^{*} \ell^{+} \ell^{-}$, is estimated.
\end{abstract}

The 2011 Europhysics Conference on High Energy Physics-HEP 2011,

July 21-27, 2011,

Grenoble, Rhône-Alpes, France

*Speaker. 


\section{Introduction}

Exclusive rare $B \rightarrow K^{(*)} l^{+} l^{-}$and $B \rightarrow K^{*} \gamma$ decays are of a great importance for the precision tests of the FCNC transitions in the Standard Model (see e.g., the review [1]). The decay amplitude

$$
A\left(B \rightarrow K^{(*)} \ell^{+} \ell^{-}\right)=\left\langle K^{(*)} \ell^{+} \ell^{-}\left|H_{e f f}\right| B\right\rangle
$$

contains hadronic matrix elements of the operators entering the effective Hamiltonian [2]. Dominant contributions in the semileptonic and radiative decays originate from the operators $O_{9(10)}=$ $\frac{\alpha_{e m}}{2 \pi}\left[\bar{s} \gamma_{\mu}\left(1-\gamma_{5}\right) b\right] \ell \gamma^{\mu}\left(\gamma_{5}\right) \ell$, and $O_{7}=-\frac{m_{b}}{8 \pi^{2}}\left[\bar{s} \sigma_{\mu v}\left(1+\gamma_{5}\right) b\right] F^{\mu v}$, respectively. Their hadronic matrix elements are factorized and reduced to $B \rightarrow K^{(*)}$ form factors. In addition, there are more complicated hadronic matrix elements generated by a common action of the quark electromagnetic current with the current-current operators $O_{1}=\left(\bar{s}_{L} \gamma_{\mu} c_{L}\right)\left(\bar{c}_{L} \gamma^{\mu} b_{L}\right)$ and $O_{2}=\left(\bar{s}_{L}^{j} \gamma_{\mu} c_{L}^{i}\right)\left(\bar{c}_{L}^{i} \gamma^{\mu} b_{L}^{j}\right)$ or with the penguin operators. In what follows, we will shortly overview the current status of $B \rightarrow K^{(*)}$ form factors and present our results concerning one of the important nonfactorizable contributions: the soft gluon emission from the charm loop induced by the operators $O_{1,2}$ together with $c$-quark e.m. current.

\section{2. $B \rightarrow K^{(*)}$ form factors from QCD light-cone sum rules}

The method of light-cone sum rules (LCSR) employs the original idea of QCD sum rules: to match the operator-product expansion (OPE) of a certain correlation function of quark currents to a hadronic dispersion relation. In the conventional version of LCSR for heavy-to-light form factors, the non-perturbative strong interaction dynamics is absorbed in the light-cone distribution amplitudes (DA's) of the light hadrons. The latest applications of this method to $B \rightarrow \pi, K$ form factors [3] contain details and references to original works. More recently, a version of LCSR with $B$ meson light-cone DA's was used in [4] (see also [5]). The current status of the $B \rightarrow K^{(*)}$ form factors relevant for the rare radiative and semileptonic $B$ decays is summarized in the Table below taken from [6], where we use $B \rightarrow K$ form factors from LCSR with kaon DA's, whereas $B \rightarrow K^{*}$ form factors are calculated using the approach with $B$-meson DA's. The latter approach still has larger uncertainties, e.g., the gluon radiative corrections have not yet been taken into account and the uncertainty in the inverse moment of the leading two-particle $B$-meson DA remains quite large. Note that $B \rightarrow K^{*}$ form factors are not yet accessible in lattice QCD with dynamical quarks, due to the large width of $K^{*}$. The use of DA's for $K^{*}$ 's with zero widths in LCSR [7] also represents a sort of a "quenched" approximation.

The LCSR predictions for the form factors are generally valid at small and intermediate momentum transfer $q^{2}$ to the lepton pair. To extrapolate these predictions to larger $q^{2}$, a series expansion based on general analytical properties of the form factors was used (the version suggested in [8]). A systematic analysis of series expansion for $B \rightarrow K^{(*)}$ form factors was performed in [9].

\section{Charm-loop effect in $B \rightarrow K^{(*)} \ell^{+} \ell^{-}$and $B \rightarrow K^{*} \gamma$}

The charm-loop effect in $B \rightarrow K^{(*)} \ell^{+} \ell^{-}$and $B \rightarrow K^{*} \gamma$ is described by a non-local hadronic 
matrix element [6]

$$
\mathscr{H}_{\mu}^{\left(B \rightarrow K^{(*)}\right)}(p, q)=i \int d^{4} x e^{i q \cdot x}\left\langle K^{(*)}(p)\left|T\left\{\bar{c}(x) \gamma_{\mu} c(x),\left[C_{1} O_{1}(0)+C_{2} O_{2}(0)\right]\right\}\right| B(p+q)\right\rangle
$$

At $q^{2} \ll 4 m_{c}^{2}$ the time-ordered operator-product receives the dominant contribution near the lightcone, $x^{2} \sim 0$. Contracting the $c$-quark fields in the propagators, one can systematically derive an OPE, which in the leading order corresponds to the factorizable charm loop. The soft-gluon emission is parameterized by the effective nonlocal quark-antiquark-gluon operator. In [6] the new nonfactorizable hadronic matrix element for this operator was computed using LCSR with threeparticle $B$-meson DA's. It is convenient to represent the charm-loop effect in $B \rightarrow K^{(*)} \ell^{+} \ell^{-}$as a process- and $q^{2}$-dependent correction to the Wilson coefficient $C_{9}$ of the operator $O_{9}$. According to our numerical estimates [6], in $B \rightarrow K \ell^{+} \ell^{-}$the nonfactorizable charm-loop amplitude at $q^{2} \ll 4 m_{c}^{2}$ amounts to several percent of the factorizable one and has a different sign. The soft-gluon effect is more pronounced in $B \rightarrow K^{*} \ell^{+} \ell^{-}$at small $q^{2}$. In $B \rightarrow K^{*} \gamma$ the factorizable charm loop contribution is absent and the nonfactorizable charm-loop effect can be expressed as a few percent correction to the Wilson coefficient $C_{7}$.

In order to access the region of large momentum transfers $\left(q^{2} \sim 4 m_{c}^{2}\right.$ and above $)$ where the light-cone OPE for the charm-loop effect breaks down, we employ hadronic dispersion relation in the variable $q^{2}$ and saturate it by $J / \psi$ and $\psi(2 S)$ contributions parameterizing the higher states by an effective pole. The influence of charm-loop contribution on the observables of $B \rightarrow K^{*} \ell^{+} \ell^{-}$is shown in Fig. 1. The correction to the differential width is quite moderate, in particular there is only a small shift of the zero-point in the forward-backward asymmetry in $B \rightarrow K^{*} \ell^{+} \ell^{-}$.

To complete the estimate of nonfactorizable effects in $B \rightarrow K^{(*)} \ell^{+} \ell^{-}$we plan to include the hard-gluon perturbative corrections to the charm-loop amplitude in this framework. Previously, this part of the effect was included in the analysis of $B \rightarrow K^{*} \ell^{+} \ell^{-}$done in [10] in terms of QCD factorization. Together with an estimate of the weak annihilation effect in these decays, a complete

\begin{tabular}{|c|c|c|c|c|}
\hline form factor & $F_{B K^{* *}}^{i}(0)$ & $b_{1}^{i}$ & $B_{s}\left(J^{P}\right)$ & input at $q^{2}<12 \mathrm{GeV}^{2}$ \\
\hline$f_{B K}^{+}$ & $0.34_{-0.02}^{+0.05}$ & $-2.1_{-1.6}^{+0.9}$ & $B_{s}^{*}\left(1^{-}\right)$ & \\
$f_{B K}^{0}$ & $0.34_{-0.02}^{+0.05}$ & $-4.3_{-0.9}^{+0.8}$ & no pole & LCSR \\
$f_{B K}^{T}$ & $0.39_{-0.03}^{+0.05}$ & $-2.2_{-2.00}^{+1.0}$ & $B_{s}^{*}\left(1^{-}\right)$ & with $K$ DA's \\
\hline$V^{B K^{*}}$ & $0.36_{-0.12}^{+0.23}$ & $-4.8_{-0.4}^{+0.8}$ & $B_{s}^{*}\left(1^{-}\right)$ & \\
$A_{1}^{B K^{*}}$ & $0.25_{-0.10}^{+0.16}$ & $0.34_{-0.80}^{+0.86}$ & $B_{s}\left(1^{+}\right)$ & \\
$A_{2}^{B K^{*}}$ & $0.23_{-0.10}^{+0.19}$ & $-0.85_{-1.35}^{+2.88}$ & $B_{s}\left(1^{+}\right)$ & LCSR \\
$A_{0}^{B K^{*}}$ & $0.29_{-0.07}^{+0.10}$ & $-18.2_{-3.0}^{+1.3}$ & $B_{s}\left(0^{-}\right)$ & with $B$ DA's \\
$T_{1}^{B K^{*}}$ & $0.31_{-0.10}^{+0.18}$ & $-4.6_{-0.41}^{+0.81}$ & $B_{s}^{*}\left(1^{-}\right)$ & \\
$T_{2}^{B K^{*}}$ & $0.31_{-0.10}^{+0.18}$ & $-3.2_{-2.2}^{+2.1}$ & $B_{s}\left(1^{+}\right)$ & \\
$T_{3}^{B K^{*}}$ & $0.22_{-0.10}^{+0.17}$ & $-10.3_{-3.1}^{+2.5}$ & $B_{s}\left(1^{+}\right)$ & \\
\hline
\end{tabular}



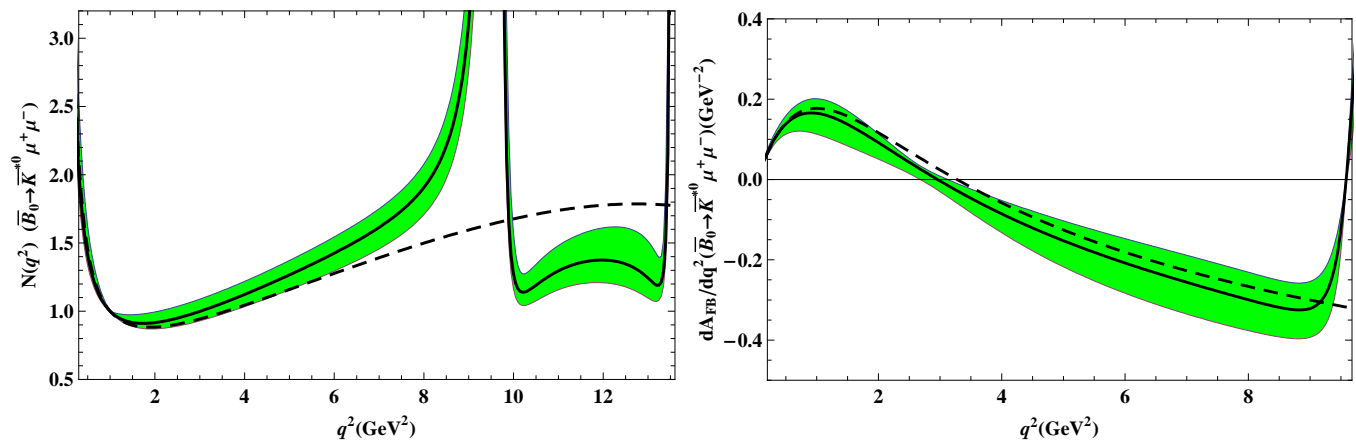

Figure 1: Differential width (left) and forward-backward asymmetry (right) of $\bar{B}_{0} \rightarrow \bar{K}^{*} \mu^{+} \mu^{-}$calculated in [6], with the charm-loop effect (solid, the shaded area indicates estimated uncertainties) and without this effect (dashed).

and accurate account of nonfactorizable effects will be achieved. This will allow to improve the accuracy of the observables of $B \rightarrow K^{(*)} \ell^{+} \ell^{-}$at low and intermediate $q^{2}$.

\section{Conclusions}

We applied the QCD sum rule methods to calculate the hadronic matrix elements involved in $B \rightarrow K^{(*)} \ell^{+} \ell^{-}$decays. The $B \rightarrow K^{(*)}$ form factors are obtained from LCSR, and the hadronic matrix elements responsible for the charm-loop effect are then calculated with the same method. In addition to the leading-order factorizable $c$-quark loop, the soft-gluon effect is taken into account and expressed in terms of the hadronic matrix element of a nonlocal operator. The influence of these effects on the decay observables was investigated, and a small shift of the zero-point of the forward-backward asymmetry in $B \rightarrow K^{*} \ell^{+} \ell^{-}$is predicted.

We are grateful to Thomas Mannel and Alexey Pivovarov for a fruitful collaboration.

\section{References}

[1] M. Artuso et al., "B, D and K decays," Eur. Phys. J. C 57 (2008).

[2] G. Buchalla, A. J. Buras and M. E. Lautenbacher, Rev. Mod. Phys. 68 (1996) 1125. Eur. Phys. J. C41, 1-131 (2005), http://ckmfitter.in2p3.fr .

[3] G. Duplancic, A. Khodjamirian, T. Mannel, B. Melic and N. Offen, JHEP 0804 (2008) 014;

G. Duplancic and B. Melic, Phys. Rev. D 78 (2008) 054015.

[4] A. Khodjamirian, T. Mannel and N. Offen, Phys. Lett. B 620 (2005) 52; A. Khodjamirian, T. Mannel and N. Offen, Phys. Rev. D 75 (2007) 054013.

[5] F. De Fazio, T. Feldmann, T. Hurth, Nucl. Phys. $B 733$ (2006) 1; JHEP 0802 (2008) 031.

[6] A. Khodjamirian, T. Mannel, A. A. Pivovarov and Y. M. Wang, JHEP 1009, 089 (2010).

[7] P. Ball and R. Zwicky, Phys. Rev. D 71, 014029 (2005).

[8] C. Bourrely, I. Caprini and L. Lellouch, Phys. Rev. D 79, 013008 (2009) [Erratum-ibid. D 82, 099902 (2010)].

[9] A. Bharucha, T. Feldmann and M. Wick, JHEP 1009, 090 (2010).

[10] M. Beneke, T. Feldmann and D. Seidel, Nucl. Phys. B 612 (2001) 25. 